\title{
Calidad en el servicio: oportunidad para el sector automotor en el Ecuador
}

\section{Quality in the service: opportunity for the automotive sector in Ecuador}

Flavio Roberto Arroyo Morocho

Carlos Santiago Buenaño Armas

Universidad Central del Ecuador, Ecuador

Autor para correspondencia: frarroyo@uce.edu.ec, sbuenanio@uce.edu.ec Fecha de recepción: 23 de Mayo de 2017 - Fecha de aceptación: 15 de Septiembre de 2017

Resumen: El sector automotor en el Ecuador ha mantenido históricamente una participación importante en la economía del país debido a los ingresos que genera en todas las actividades económicas directas e indirectas que involucra. A partir de la industria automotriz se han impulsado a otras industrias como la siderúrgica, metalúrgica, metalmecánica, minera, petrolera, petroquímica, del plástico, vidrio, entre otras; industrias claves para la manufactura de vehículos. El sector automotor integra a diferentes actores, como el caso de las empresas autopartistas, proveedoras de partes y piezas; por otra parte, las ensambladoras de vehículos han sido quienes impongan los estándares productivos en la cadena de producción. Actualmente, existe una ardua competencia entre las empresas por ser el primero en obtener la atención del cliente. La calidad en el servicio es uno de los puntos principales que se deben cumplir dentro de cada una de las empresas. Una correcta gestión en la atención al cliente es la mejor estrategia que se puede utilizar para lograr una empresa de éxito y de esa manera poder sobrellevar períodos complicados como el que atraviesa el sector automotor del Ecuador en la actualidad.

Palabras Clave: calidad; servicio; productividad; postventa; industria automotriz; sector automotor; cliente

Abstract: The Ecuadorian sector in the Ecuador has historically kept an important participation in the economy of the country because to incomes that generate in all direct and indirect economic activities that involve. From the automotive industry have been propelled to other industries such as steel, metallurgical, mining, oil, petrochemical, plastic, glass, among others; key industries for the vehicle manufacturing. The automotive sector integrates to different actors, such as the case of the autoparts companies, parts and accessories suppliers; too, the assemblers of vehicles have been who that impose the production standards in the production chain. There is arduous competition between the companies for being the first to obtain the customer's attention. The quality of service is one of the main points that must be comply within each one of the countries. A correct management in the customer's attention is the best strategy that can be used for reaching a successful company and in this way, can cope complicated periods as the one that crosses the automotive sector of Ecuador at present.

Key words: quality; service; productivity; after-sales service; automotive industry; automotive sector; customer 


\section{Introducción}

A nivel mundial la industria automotriz cumple un papel importante en el desarrollo económico de las naciones; se ha constituido como precursora de competitividad e innovación, ha evolucionado de la mano con la tecnología, de los nuevos materiales y de procesos de manufactura más eficientes. Las principales compañías de diseño y manufactura de vehículos, día a día deben innovar en nuevos modelos y prestaciones de los vehículos para atraer a nuevos clientes y fidelizar a sus clientes tradicionales. En la actualidad los vehículos deben ofrecer diversas características que llamen la atención del cliente más allá de lo referente a seguridad y confort.

Ahora bien, las empresas manufactureras no deben centrarse únicamente en la producción de nuevos vehículos. En un mundo cada vez más globalizado, el comportamiento de los mercados es muy variable; los clientes cuentan con mayores opciones de compra ocasionando que estos se vuelvan más exigentes no sólo con el vehículo adquirir, sino también con la calidad del servicio que se pretende recibir.

Cuando se vende un vehículo, la marca adquiere una obligación ética en ese bien y con su cliente; ya que, la marca debe ser responsable de todo el proceso del servicio al cliente, que inicia la oferta y venta, el servicio postventa que engloba los subprocesos de mantenimiento preventivo y correctivo, y en la actualidad ofrecer facilidades en la renovación del vehículo. Todo este proceso constituye una moderna forma de mejorar la competitividad del sector automotor, fundamentando en la calidad en el servicio como la variable crítica para ser más competitivos en el mercado.

En el Ecuador, la industria automotriz ha generado gran desarrollo tecnológico en los últimos 30 años, dado que contribuye al desarrollo nacional con inversiones, capacitación, tecnología y generación de divisas. Las ensambladoras en el país, debido a su alto grado de tecnificación, son reconocidas por sus productos de alta calidad en el mercado nacional e internacional (Pro Ecuador, 2017).

El sector automotor tiene una participación importante en la economía del país debido a los ingresos que genera en todas las actividades económicas directas e indirectas que involucra. Se debe destacar que la industria automotriz ha impulsado a otras industrias del sector productivo como la siderúrgica, metalúrgica, metalmecánica, minera, petrolera, petroquímica, del plástico, vidrio, electricidad, robótica e informática, industrias claves para la elaboración de los vehículos. De este modo, el sector automotor integra a diferentes actores, tanto para las firmas autopartistas proveedoras de partes y piezas; así como para las ensambladoras que son las firmas que imponen los estándares productivos de la cadena (Pro Ecuador, 2017)

El ensamblaje de vehículos en el Ecuador se inició en 1973, siendo AYMESA Autos y Máquinas del Ecuador S.A. la empresa pionera en esta actividad, con la producción de un vehículo de transporte básico diseñado por la General Motors "El Andino". La entrada al mercado de ensamblaje por AYMESA fue seguida por otras empresas ensambladoras: MARESA en 1975 y ÓMNIBUS BB en 1976 empresa que comenzó siendo una empresa rectificadora de cigüeñales (Laso Rivadeneira, 2001). 
Con posterioridad se creó una cuarta empresa ensambladora COENANSA de vehículos FIAT cuyo asiento fue la provincia de Manabí. Esta empresa pertenecía al "Grupo Noboa" y tuvo una vida muy corta pues comenzó sus operaciones en 1992 y las finalizó en 1996 (Laso Rivadeneira, 2001).

En febrero de 2012 se instaló la ensambladora Ciudad del Auto (CIAUTO) en Ambato, siendo la cuarta del país. En este lugar se producen los modelos Haval H5 y la camioneta Wingle de la empresa Great Wall. Por otro lado, la empresa Thunder Cycles en el 2012 se convirtió en la primera y única marca de motos ensambladas $100 \%$ en Ecuador. La alianza estratégica entre Metaltronic (fabricante de autopartes para la industria automotriz) y Thunder Cycles en Ecuador (ensambladora de motocicletas) (Pro Ecuador, 2017).

La industria y ensamblaje en el Ecuador han permitido el desarrollo de la producción local de componentes, partes, piezas e insumos, creando un encadenamiento productivo en la fabricación de otros productos relacionados a los automotrices, maquinarias y herramientas.

A escala nacional, de acuerdo a la información del Censo Nacional Económico 2010, existen 29,068 establecimientos económicos dedicados a actividades de comercio automotriz, de los cuales el $70 \%$ corresponde a establecimientos que realizan mantenimiento y reparación de vehículos automotrices, mientras que el $30 \%$ restante se dedica a la venta de partes, piezas y accesorios de vehículos; venta al por menor de combustibles y venta de vehículos (Pro Ecuador, 2017).

Por otro lado, existen 118 empresas dedicadas a prestar servicios de alquiler de vehículos en todo el país, lo que representa el $0.40 \%$ del número total de establecimientos contemplados dentro de las actividades del sector automotor (Pro Ecuador, 2017).

Las actividades relacionadas al sector automotor generan un importante número de plazas de empleo. De acuerdo a información del Censo Económico 2010 se tienen 90,012 personas ocupadas, de las cuales el $83 \%$ son hombres y el 17\% mujeres. En los establecimientos de comercio se encuentran ocupadas 84,155 personas, en manufactura 5,194 y en servicios 663 (Pro Ecuador, 2017).

Las transformaciones de la industria automovilística en las últimas décadas están alterando este mercado haciendo que la postventa desempeñe un papel esencial en las estrategias del sector automóvil. El papel de los fabricantes de automóvil en la distribución y postventa del vehículo, las nuevas formas de distribución de repuestos, las nuevas relaciones entre las marcas y los concesionarios y talleres autorizados, en definitiva, la estructura de la cadena aguas abajo son algunos de los aspectos competitivos más interesantes de analizar en este sector (Rastrollo \& Martínez, 2004).

El servicio post venta consiste en la atención al cliente posterior a la compra de un vehículo. El servicio post venta es una de las mejores formas de generar lealtad hacia una marca y conseguir la fidelidad de sus clientes. Un cliente satisfecho es la mejor publicidad que se puede tener, ya que incentiva el marketing de referencia. 
Indudablemente de uno de los factores que hacen un cliente esté satisfecho con su automóvil es la calidad del servicio que recibe cada vez que acude al taller del concesionario.

El mercado postventa del automóvil es importante tanto en términos cuantitativos como cualitativos, lo cual refleja en qué medida los servicios están adquiriendo protagonismo en la oferta global de los fabricantes industriales. El servicio postventa es un área de desarrollo clave para la capacidad competitiva del conjunto de agentes de la cadena (Rastrollo \& Martínez, 2004).

\section{Metodología}

\section{Calidad en el Servicio}

La calidad, el servicio y la calidad en el servicio se han convertido en los últimos años en la principal estrategia de diferenciación entre las organizaciones de clase mundial. L. M. Huete (1998) dice que es muy difícil establecer líneas de frontera entre la calidad y el servicio, es decir, que no hay una línea dividida. La intención de incorporar la calidad en el servicio dentro de la gestión diaria del negocio se aprecia cada vez más en el contexto empresarial. La calidad y el servicio son dimensiones que están presentes de manera articulada para beneficio y satisfacción de las necesidades del hombre (Vargas \& Aldana de Vega, 2015).

El servicio, sin duda alguna como la calidad, acompaña al hombre en todo su quehacer desde el inicio de la vida. Sin embargo, fue en los años 80 cuando e declaró como una estrategia empresarial que da valor agregado a las organizaciones (Vargas \& Aldana de Vega, 2015). No se puede desconocer el desarrollo de nuevos modelos alrededor de la calidad en el servicio que han permitido de una manera sistémica los procesos, las actividades, la innovación y la creatividad.

Calidad en el servicio es el hábito desarrollado y practicado por una organización para interpretar las necesidades y expectativas de sus clientes y ofrecerles, en consecuencia, un servicio accesible, adecuado, ágil, flexible, apreciable, útil, oportuno, seguro y confiable, aún bajo situaciones imprevistas o ante errores, de tal manera que el cliente se sienta comprendido, atendido y servido personalmente, con dedicación y eficacia, y sorprendido con mayor valor al esperado, proporcionando en consecuencia mayores ingresos y menores costos para la organización (Pizzo, 2013).

Desde el punto de vista del cliente la calidad en el servicio puede definirse como el grado en que un servicio cumple con las expectativas, deseos y demandas de sus clientes. El juicio que el cliente realiza sobre la excelencia del servicio recibido es subjetivo, Por tanto el nivel de calidad de los servicios prestados dependerá por una parte de comprender las necesidades del cliente y por otra parte la capacidad de la organización con que se lleve el proceso.

En una empresa que trabaja respetando la filosofía de la calidad de servicio, el protagonista principal es el cliente. En este caso, la empresa tiene como objetivo fundamental eliminar de forma permanente todos aquellos problemas, errores o equivocaciones que pueda generar la insatisfacción del cliente. Lo más importante es satisfacer sus necesidades e incluso exceder sus expectativas (Pérez, 2013). 
La calidad en el servicio se puede definir como el grado de satisfacción del cliente, basado en la comparación entre lo que esperaba recibir, y lo que su percepción le indica que ha recibido. La evaluación de la calidad en el servicio se la realiza sobre la totalidad del servicio o el servicio completo una vez finalizado, de tal manera que lo agradable del servicio depende de la calidad de todo el proceso, esto permitirá que una empresa pase de ser objeto anónimo, a tener un rostro conocido.

En In Search of Excellence, Peters y Waterman dieron nuevo respaldo a las empresas orientadas hacia la "satisfacción del cliente". Las primeras investigaciones acerca de la satisfacción del cliente señalaron que tanto los clientes satisfechos como los que no lo están influyen de manera decisiva sobre el desempeño básico de la empresa. Cien clientes satisfechos producen 25 nuevos clientes. Por cada queja recibida, existen otros 20 clientes que opinan lo mismo pero que no se molestan en presentar queja y finalmente el coste de conseguir un nuevo cliente equivale a cinco veces el de mantener satisfecho al que ya está ganado (Dutka, 1998).

\section{Técnicas y Herramientas}

Hoy en día con la creciente globalización las empresas líderes están más preocupadas por saber cómo mantenerse con clientes más exigentes, de ahí la necesidad de implementar herramientas que satisfagan no solo a sus clientes, sino también a sus propios objetivos empresariales y a sus expectativas de mejora de sus indicadores de productividad. Estas técnicas y herramientas, son procedimientos formalizados que ayudan a las empresas a medir la calidad de sus servicios y a planificar la mejora de sus procesos.

Los Círculos de Calidad propician la integración y el involucramiento del personal de la empresa con el objetivo de mejorar, ya sea productos o procesos. Permiten que los trabajadores y la administración compartan la responsabilidad de definir y resolver problemas de coordinación, productividad y por supuesto de calidad.

El Diagrama Causa Efecto es una herramienta que ilustra gráficamente las relaciones entre un efecto (resultado) y sus causas (factores), permitiendo identificar, clasificar y evidenciar posibles causas, tanto de problemas específicos como de características de calidad en el servicio prestado. Estimula la participación en grupos, con lo que se obtiene mayor provecho de los conocimientos individuales de cada miembro sobre el proceso.

El Diagrama de Pareto puede determinar la causa clave de un problema, aislándola de otras de menor importancia, y contrastar la efectividad de las mejoras obtenidas comparándolas con diagramas de distintos momentos. El Diagrama de Pareto puede utilizarse para investigar tanto efectos como causas.

La Lluvia o Tormenta de Ideas (brainstorming), permite producir ideas en equipo, progresivamente superiores y más completas o amplias, sobre los problemas de un área, las causas de los mismos, o sobre las soluciones a los problemas.

Los Diagramas de Flujo describen gráficamente el funcionamiento y la estructura de los procesos de una empresa, sus fases y relaciones entre sus componentes, ofreciendo una visión 
global de todos ellos. Mediante los diagramas de flujo se puede identificar claramente un proceso, describiendo el trayecto que sigue un producto o servicio, así como responsables y recursos que lo constituyen.

Los Histogramas permiten evaluar la eficiencia de las medidas de mejoras implantadas o para evidenciar el grado de cumplimiento de las especificaciones de los límites determinados en los diferentes procesos de la empresa.

El Control Estadístico de Procesos (CEP), es un conjunto de herramientas estadísticas que permiten recopilar, estudiar y analizar la información de procesos repetitivos para poder tomar decisiones encaminadas a la mejora de la calidad de los productos, así como la mejora de estos, es aplicable tanto a procesos productivos como de servicios

El Análisis Modal de Fallos y Efectos - AMEF, es una herramienta que permite identificar las variables significativas de un proceso o producto para poder establecer las acciones correctoras necesarias, con lo que se previenen los posibles fallos y se evita que lleguen al cliente productos defectuosos. La metodología AMEF está orientada a maximizar la satisfacción del cliente gracias a la eliminación o minimización de posibles problemas.

Benchmarking es un proceso continuo que consiste en comparar y medir los procesos internos de una organización con los de otra con mejores resultados. Es una herramienta para mejorar las prácticas de un negocio y, por ende, su competitividad.

Despliegue Funcional de la Calidad (Quality Function Deployment - QFD), es una metodología que permite identificar y trasladar la información obtenida del cliente y convertirla en requisito del producto. Las expectativas del cliente serán las que marquen todo el proceso creativo: se debe de traducir la "voz del cliente" al lenguaje técnico.

Seis Sigma es una metodología de mejora de procesos, permite una reducción drástica de los defectos en el producto a través del seguimiento diario de todas las actividades de la empresa, que permite minimizar sus desperdicios e incrementar la satisfacción de los clientes, enfocándose en las necesidades y requerimientos.

Con el Cuadro de Mando Integral (Balanced Scorecard - BSC) se reorienta el sistema gerencial y se enlaza la estrategia a corto y a largo plazo, permite detectar de forma automática desviaciones en el plan estratégico u operativo, incluso indagar en los datos operativos de la compañía hasta descubrir la causa original de esas desviaciones.

\section{Resultados}

El sector automotor ecuatoriano se había caracterizado hace algunos años por un crecimiento en ventas y unidades constantes cada año. Sin embargo, las nuevas leyes y medidas restrictivas han favorecido a un nuevo escenario donde no es posible seguir creciendo dadas las limitaciones del sector (Business cost management, 2016). Existen tres tipos de actividades que se realizan en el sector automotor: Comercialización, Manufactura y Servicios. 
Según el censo realizado por el INEC para el año 2014 existían 29.068 establecimientos que se dedican a actividades relacionadas con el comercio automotriz, $70 \%$ dedicados al mantenimiento y reparación de vehículos y el 30\% venta de partes, piezas y accesorios de vehículos y venta al por menor de combustibles y vehículos. Dentro de este sector mayoritario se encuentran los establecimientos como concesionarios, talleres automotrices, establecimientos de venta de repuestos, llanteras, establecimientos de compra venta de vehículos nuevos y usados, entre otros (Business cost management, 2016).

Según cifras de la Asociación de Empresas Automotrices del Ecuador (AEADE), las empresas ensambladoras produjeron 26.786 vehículos en el año 2016, como se puede ver en la figura 1 .

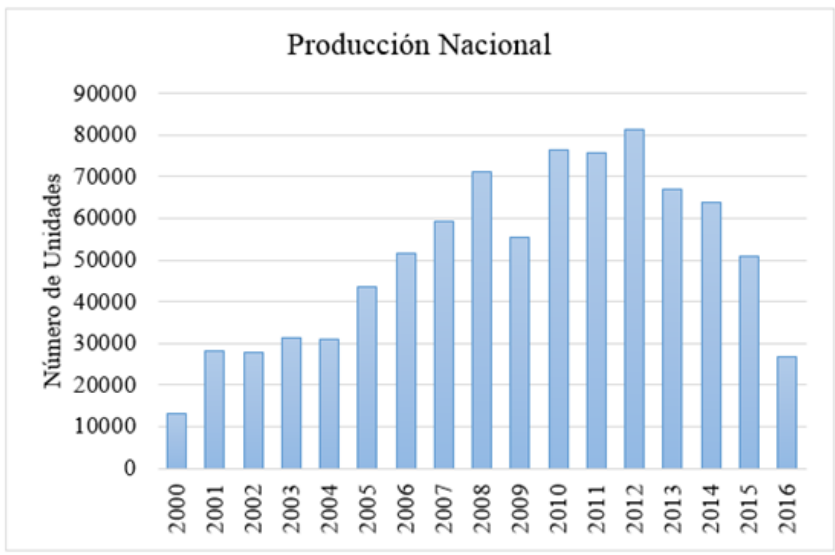

Figura 1. Produccion nacional de vehículos Fuente: Anuario 2016 (AEADE, 2017)

Como se puede observar en la figura 2, las exportaciones del sector automotor en el 2016 fueron de 716 unidades, siendo el año más bajo del período 2000 - 2016; esta reducción se debe principalmente a la resolución 50 del Comité de Comercio Exterior emitida el 20 de diciembre del 2015, que entró en vigencia el 1 de enero del 2016, en donde se estableció una cuota global para las importaciones de vehículos terminados y para ensamblaje en el país.

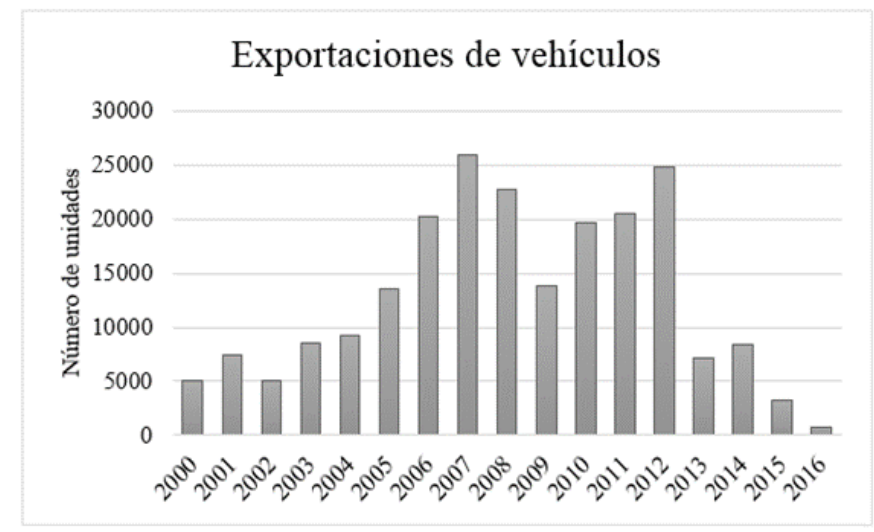

Figura 2. Exportación nacional de vehículos Fuente: Anuario 2016 (AEADE, 2017) 
El decrecimiento dela importaciones de vehículos se reflea con mayor evidente en el año 2016 cuando se importaron tan solo 31775 unidades, debido a la resolución 50 del Comité de Comercio Exterior del 30 de diciembre del 2015, en donde se estableció una cuota global para las importaciones de vehículos terminados como se indicó anteriormente.

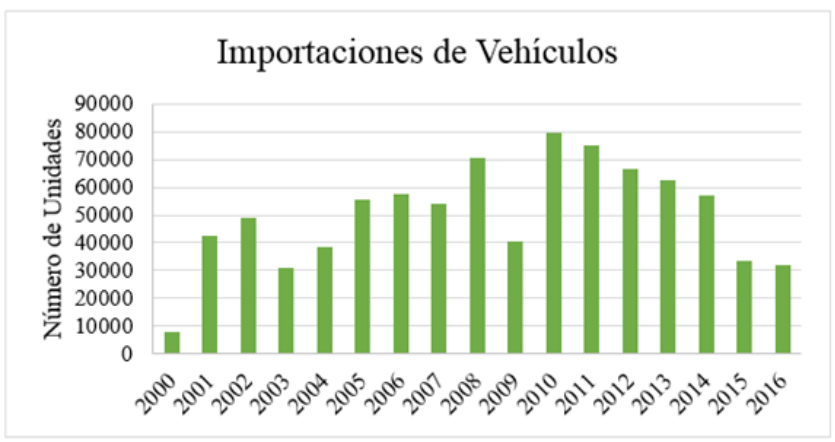

Figura 3. Importación nacional de vehículos Fuente: Anuario 2016 (AEADE, 2017)

El año 2016 inicia con una reducción del 32\% en las ventas de vehículos con respecto al 2015, disminución que se responsabiliza a la contracción económica que vivió el país y a la redujeron los cupos para las unidades importadas en cerca del $40 \%$ y para los $\mathrm{CKD}^{1}$ de ensamblaje local en un 20\%. Las ventas totales de vehículos en Ecuador en el año 2016 alcanzaron las 63.555 unidades valor inferior en un 22\% en relación al año anterior.

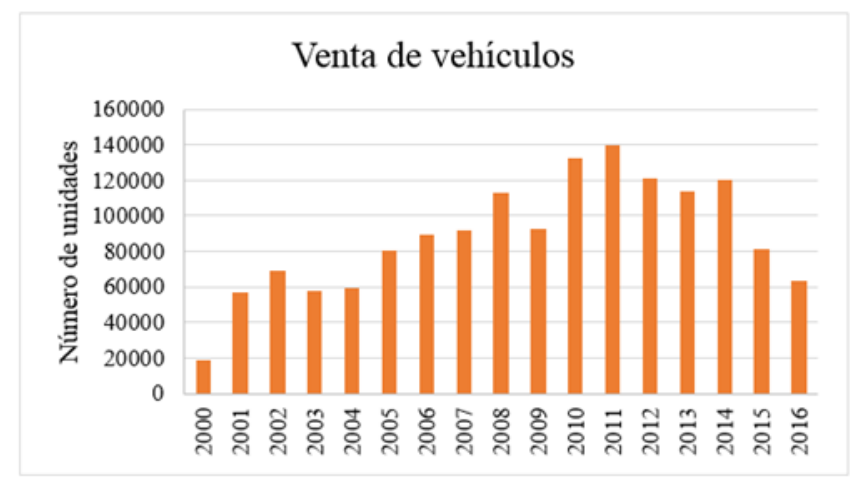

Figura 3. Importación nacional de vehículos Fuente: Anuario 2016 (AEADE, 2017)

Como se puede observar durante los últimos años el sector automotor ha sido afectado repetidamente. Las restricciones a las importaciones de vehículos y sus partes han hecho que la industria automotriz se enfrente a un escenario nuevo y se vea obligada a considerar alternativas en el negocio que le permitan mantenerse mientras llegan expectativas positivas que le permitan desarrollarse y volver a ser un sector importante en la economía nacional.

Este contexto nos permite establecer alternativas que sean factibles para evitar que el sector automotor se encuentre inmerso en una crisis financiera y que los sectores vinculados a esta industria mantengan niveles de rendimientos económicos no tan desfavorables.

\footnotetext{
1 “Completly Knock Down” (Kit de montaje)
} 


\section{Discusión}

Como se puede evidenciar la situación del sector automotor no es favorable, restricciones, incrementos arancelarios, la imposición de cupos para importar autos o componentes han ocasionado que la industria decaiga. Las restricciones gubernamentales incidieron en el comportamiento de las ventas del sector.

Las empresas del sector automotor ecuatoriano deben potenciar los servicios postventa, como medida para reducir el impacto económico producido por el descenso de las ventas de vehículos. Es importante ofrecer a sus clientes un adecuado servicio postventa; ya que, se genera lealtad y fidelidad hacia su empresa y por ende a la marca.

El servicio postventa es un área de desarrollo clave para la capacidad competitiva de las empresas. El fabricante establece su red oficial de concesionarios exclusivos, a quienes confiere la responsabilidad de postventa en talleres autorizados. Los talleres autorizados son, por regla general, establecimientos correctamente acondicionados para la prestación de servicios de mantenimiento preventivo y correctivo de los vehículos de la marca que representan, cuentan con equipos y herramientas adecuadas y con personal capacitado.

Por otra parte, los talleres independientes, que es un subsector muy fragmentado formado por una gran variedad de empresas o talleres de reducidas dimensiones con un desigual acceso a inversiones en equipamiento, herramientas e información y sistemas y tecnologías de diagnóstico. A pesar de esto, este subsector no está limitado a tareas de mantenimiento preventivo, sino que atiende a un amplio porcentaje del parque automotor y ofrece una gama completa de servicios.

La diferenciación del producto obliga a las empresas a innovar en el servicio ofrecido, se debe pensar en una satisfacción que incluyen todos aquellos servicios relacionados con la adquisición y uso del mismo tales como servicios de mantenimiento, reparación, venta de accesorios y repuestos, servicios financieros y seguros.

La calidad en el servicio es uno de los puntos clave para permanecer entre la preferencia de los consumidores, siendo a su vez un diferenciador ante la competencia, y es el punto decisivo en el cual dichos clientes se crean una opinión positiva o negativa sobre la organización.

La calidad en el servicio permite cumplir con las expectativas y necesidades de cada uno de nuestros clientes. Cuando una empresa conoce con precisión la clase de servicio que sus clientes esperan, se puede decir que ofrece un excelente servicio a su cliente y por ende una ventaja competitiva.

La calidad del servicio que se presta al cliente es un valor agregado invisible que puede determinar que aquel vuelva o que nunca más regrese a nuestro negocio. La revolución del servicio es un fenómeno de cada vez mayor impacto en las economías modernas, se puede determinar que todas las empresas consideran de importancia la calidad del servicio como factor clave para mejoramiento de su productividad. 


\section{Conclusiones}

El sector automotor en el Ecuador ha sido sin duda uno de las afectadas por la situación económica del país. Las bajas en la producción y la caída de ventas sustentan esta realidad. La industria automotriz entre los años 2010 y 2012 registró un significativo incremento en su producción ensamblando un promedio de 78560 vehículos, años con los valores más altos de producción, panorama distinto se vivió en el año 2013 en donde la producción de vehículos ensamblados disminuyó a 66844 vehículos. Desde este año la producción ha seguido a la baja llegando a las 26786 unidades ensambladas en el país en el año 2016.

La disminución en el ensamblaje de vehículos en Ecuador no solo se refleja en las ventas bajas de las diferentes empresas, sino que hay una afectación en la cadena de producción nacional. Las empresas proveedoras más conocidas como autopartistas que entregan sus productos a las ensambladoras, reciben menos pedidos por la falta de producción, ocasionado recortes de personal y en algunas ocasiones el cierre definitivo del negocio.

El servicio postventa se presenta como una alternativa de creciente valor y rentabilidad en el conjunto de este sector, y además constituye un escalón básico para asegurar la capacidad de relación con el cliente por parte de las empresas comercializadoras de automóviles en Ecuador.

En la actualidad existen una gran competencia entre empresas del sector, es importante ampliar ventajas competitivas para lograr mantener niveles adecuados de productividad; la calidad en el servicio es una de esas ventajas que se pueden desarrollar fácilmente, siempre y cuando se implementen de forma correcta técnicas o herramientas que permitan sobrepasar las expectativas del cliente.

La calidad en el servicio en las empresas vinculadas al sector automotor es uno de los puntos clave para permanecer entre la preferencia de los consumidores, ya sea como comercializadoras de vehículos o como empresa autopartista. Un servicio postventa de calidad permite mejorar la imagen que se proyecta al mercado; siendo a su vez un diferenciador ante la competencia, y es el punto decisivo en el cual los clientes se crean una opinión positiva sobre la empresa.

\section{Bibliografía}

AEADE. (2017). Anuario 2016. Quito: Asociación de Empresas Automotrices del Ecuador.

Business cost management. (2016). Reducción de costos en tiempos de crisis/Evolución del Sector automotriz Ecuatoriano . Quito: BCM.

Dutka, A. F. (1998). Manual de AMA para la satisfacción del cliente. Buenos Aires: Ediciones Granica S.A.

Laso Rivadeneira, M. (2001). La Industria Automotriz Ecuatoriana frente a la Apertura Comercial. Quito: Universidad Andina Simón Bolivar. 
Pérez, V. (2013). Calidad Total en la Atención Al Cliente. Vigo: Ideaspropias Editorial S.L.

Pizzo, M. (29 de Agosto de 2013). Cómo servir con exelencia. Obtenido de http://comoservirconexcelencia.com/blog/construyendo-una-definicion-de-calidad-en-elservicio/.html

Pro Ecuador. (2017). Análisis sectorial Automotriz 2017. Quito: Instituto de Promoción de Exportaciones e Inversiones.

Rastrollo, M. Á., \& Martínez, P. (2004). El mercado de servicios postventa del automóvil. Tendencias hacia un nuevo modelo de organización. Boletín ICE Económico, 17-29.

Vargas, M., \& Aldana de Vega, L. (2015). Calidad y servicio: Conceptos y herramientas. Bogotá: Ecoe Ediciones. 